\title{
Studies on Synthesis of Some Novel Heterocyclic Chalcone, Pyrazoline, Pyrimidine - 2 - One, Pyrimidine - 2 - Thione, para-Acetanilide Sulphonyl and Benzoyl Derivatives and their Antimicrobial Activity
}

\author{
RAKESH N. MISTRY AND K. R. DESAI* \\ Department of Chemistry, \\ Veer Narmad South Gujarat University, Surat - 395 007, India. \\ bisheshbulsara@hotmail.com
}

Received 5 November 2004; Accepted 14 December 2004

\begin{abstract}
Dichloro benzene on chlorosulphonation by chlorosulphonic acid gives 1, 2 - [dichloro] - benzene sulphonyl chloride which on condensation with $\mathrm{p}$ -amino acetophenone gives 1-[acetyl] - 1' , 2' - [dichloro] - dibenz sulphonamide derivative. This derivative undergo condensation with 2,4- dichloro benzaldehyde gives 1- [3" - (sub. phenyl) - 2" - propene - 1" - one] - 1' , 2' - [dichloro] - dibenz sulphonamide derivative which on reaction with $99 \%$ hydrazine hydrate and glacial acetic acid gives 1-[acetyl]-3- [1' , 2' - (dichloro) - dibenz sulphonamide] -5 - [2", 4" - dichloro phenyl] - 2 - pyrazoline derivative. This derivative reacts with various substituted aldehydes to give corresponding substituted chalcone derivatives $[1(\mathrm{a}-\mathrm{j})]$. Now, these chalcone derivatives $[1(\mathrm{a}-\mathrm{j})]$ on condensation with urea gives corresponding substituted pyrimidine - 2 - one derivatives [2(a-j)] and on condensation with thio-urea gives corresponding substituted pyrimidine- 2 -thione derivatives [3(a-j)]. Further, these chalcone derivatives $[1(\mathrm{a}-\mathrm{j})]$ on reaction with $99 \%$ hydrazine hydrate gives 1 - [1' - (H) - 5' - (sub. phenyl) - 2' pyrazoline]- 3 - [1", 2" - (dichloro) - dibenz sulphonamide] - 5 - [2", , 4", dichloro phenyl]-2- pyrazoline derivatives [4(a-j)] as an intermediate compounds, which on condensation with p-acetanilide sulphonyl chloride gives corresponding substituted $\mathrm{p}$ - acetanilide sulphonyl derivatives [5(a-j)] and on condensation with benzoyl chloride gives corresponding substituted benzoyl derivatives [6(a-j)]. Structure elucidation of synthesised compounds has been made on the basis of elemental analysis, I.R. spectral studies and ${ }^{1}$ H N.M.R. spectral studies. The antimicrobial activity of the synthesised compounds has been studied against the cultures "Staphylococcus aureus", "Escherichia coli" and "Candela albicans".
\end{abstract}

Key words: Synthesis, Heterocyclic substituted chalcone derivatives, pyrazoline derivatives, benzoyl derivatives, antimicrobial activity. 


\section{Introduction}

The chalcones are $\alpha, \beta$ - unsaturated ketones containing the reactive ketoethylenic group $-\mathrm{CO}-\mathrm{CH}$ $=\mathrm{CH}-$. Presence of $\alpha, \beta$ - unsaturated carbonyl system in chalcone makes it biologically active. Some substituted chalcones and their derivatives have been reported to possess some interesting biological properties such as antibacterial, antifungal ${ }^{1}$, insecticidal ${ }^{2}$, anaesthetic ${ }^{3}$, analgesic, ulcerogenic ${ }^{4}$ etc.

Pyrazole belongs to the family of azoles i.e. five-membered ring containing nitrogen and carbon atom. The dihydro pyrazoles are called pyrazolines. Some substituted pyrazolines and their derivatives have been reported to possess some interesting biological activities such as anticancer ${ }^{\mathbf{5}}$, insecticidal ${ }^{\mathbf{6}}$, antibacterial $^{7}$ etc.

The replacement of two $-\mathrm{CH}$ units in benzene by nitrogen atoms gives pyrimidines. Some substituted pyrimidines and their derivatives have been reported to possess antimicrobial, antitumour and antifungal ${ }^{\mathbf{8}}$ activities. It has incidental antiviral activity against herpes and vaccinia infections ${ }^{9}$.

All these observations and the essential role of heterocyclic chalcone derivatives, pyrazoline derivatives and pyrimidine derivatives, in certain biological reactions prompted us to synthesise all these heterocyclic derivatives $[\mathbf{1}(\mathbf{a}-\mathbf{j})$ to $\mathbf{6}(\mathbf{a}-\mathbf{j})]$.

\section{Experimental}

\section{Preparation of 1, 2 - dichloro benzene sulphonyl chloride derivative}

In a $750 \mathrm{ml}$. three-necked flask, place chlorosulphonic acid $(0.01 \mathrm{~mol})$ and cool to $0^{\circ} \mathrm{c}$ in a freezing mixture of ice and salt. Introduce 1,2 - dichloro benzene $(0.01 \mathrm{~mol})$ from the dropping funnel dropwise with constant stirring at such a rate that the temperature of the well-stirred mixture does not rise above $5^{\circ} \mathrm{c}$. When all the 1,2 - dichloro benzene has been added (about 3 hours), stirr the reaction mixture for 4 hours and then allow to stand overnight in a refrigerator. Pour the resultant solution onto crushed ice, separate the aqueous solution from the oily layer and wash the latter several times by decantation with cold water. Now, cool the oil at -10 to $-20^{\circ} \mathrm{c}$ with ice and calcium chloride for several hours, the almost pure 1,2 - dichloro benzene sulphonyl chloride will crystallise out. Filter it at the pump upon a sintered glass funnel and purify it by recrystallisation from light petroleum. M. P. $118^{\circ} \mathrm{c} .$, Yield $82 \%$.

\section{Preparation of 1 - [Acetyl] - 1', 2' - [dichloro] - dibenz sulphonamide derivative}

To a solution of 1,2 - dichloro benzene sulphonyl chloride $(0.01 \mathrm{~mol})$ in benzene $(30 \mathrm{ml}), \mathrm{p}$ - amino acetophenone $(0.01 \mathrm{~mol})$ was added and the reaction mixture was refluxed on water bath at $80-90^{\circ} \mathrm{c}$ gently for about 5-6 hours. After the completion of reaction, the resultant solution was concentrated to half of its volume and cooled in an ice-bath. The solid separated was filtered, washed with hot water, dried and crystallised from ethanol. M. P. $127^{\circ} \mathrm{c}$., Yield $76 \%$.

Preparation of 1 - [3', - (2',' , 4',' -Dichloro phenyl) - 2', - propene - 1', - one] - 1' , 2' [dichloro]-dibenz sulphonamidederivative

To a well-stirred solution of 1 - [acetyl] - 1', 2' - [dichloro] - dibenz sulphonamide (0.01 mol) in ethanol $(40 \mathrm{ml})$ and $40 \%$ sodium hydroxide (condensing agent) $(25 \mathrm{ml}) ; 2,4$ - dichloro benzaldehyde $(0.01 \mathrm{~mol})$ was added slowly at $0-5^{\circ} \mathrm{c}$. After the completion of addition, the reaction mixture was stirred for further 3 hours and left overnight. The content was then poured over crushed ice and acidified with dilute $\mathrm{HCl}$. The resulting solid so obtained was filtered, washed several times with distilled water, dried and crystallised from ethanol. M. P. $132^{\circ}$ c., Yield $78 \%$. 
Preparation of 1 - [acetyl] - 3 - [1', 2' - (dichloro) - dibenz sulphonamide] - 5 - [2', , 4', dichloro phenyl] - 2 - pyrazoline derivative

To a solution of 1 - [3"' - (2',', 4', - dichloro phenyl) - 2'" - propene - 1'" - one] - 1', 2' - [dichloro] dibenz sulphonamide $(0.01 \mathrm{~mol})$ in ethanol $(30 \mathrm{ml})$, a $99 \%$ hydrazine hydrate $(0.015 \mathrm{~mol})$ was added drop-wise followed by glacial acetic acid $(10 \mathrm{ml})$ and the reaction mixture was refluxed on water bath at $70-80^{\circ} \mathrm{c}$ gently for about $8-10$ hours. After the completion of reaction, the reaction mixture was concentrated and cooled, the resulting solid so obtained was filtered, washed several times with distilled water, dried and crystallised from ethanol. M. P. $143{ }^{\circ}$ c., Yield $76 \%$.

Preparation of 1 - [3"' - (2',' - hydroxy phenyl) - 2', - propene - 1"' - one] - 3 - [1', 2' - (dichloro) dibenz sulphonamide] - 5 - [2,",', 4,",' - dichloro phenyl] - 2 - pyrazoline derivative [1(a)]

To a well-stirred solution of 1 - [acetyl] - 3 - [1', 2' - (dichloro) - dibenz sulphonamide] - 5 - [2', 4', dichloro phenyl] - 2 - pyrazoline $(0.001 \mathrm{~mol})$ in ethanol $(30 \mathrm{ml})$ and $40 \%$ sodium hydroxide (condensing agent) $(15 \mathrm{ml}) ; 2$ - hydroxy benzaldehyde $(0.001 \mathrm{~mol})$ was added slowly at $0-5^{\circ} \mathrm{c}$. After the completion of addition, the reaction mixture was stirred for further 3 hours and left overnight. The content was then poured over crushed ice and acidified with dilute HCI. The resulting solid so obtained was filtered, washed several times with distilled water, dried and crystallised from ethanol. M. P. $152^{\circ} \mathrm{c}$. , Yield $70 \%$.

Similarly, the remaining substituted chalcone derivatives $[\mathbf{1}(\mathbf{b}-\mathbf{j})]$ were prepared by the same procedure as discussed above.

Physical and Analytical data of compounds [1(a-j)] are presented in Table-1 and Antimicrobial data of compounds [1(a-j)] are presented in Table-7.

Preparation of 1 - [1"' - (h) - 6"' - (2",' -Hydroxy phenyl) - pyrimidine - 2"' - one] - 3 - [1', 2' - (dichloro) - dibenz, sulphonamide] - 5 - [2,",', 4,",' - dichloro phenyl] - 2 -pyrazoline derivative [2(a)]

A mixture of 1 - [3"' - (2',' - hydroxy phenyl) - 2', - propene - 1', - one] - 3 - [1', 2' - (dichloro) dibenz sulphonamide] - 5 - [2,", , 4,", - dichloro phenyl] - 2 - pyrazoline [1(a)] $(0.001 \mathrm{~mol})$ and urea $(0.001 \mathrm{~mol})$ was dissolved in ethanol $(30 \mathrm{ml})$. A dilute hydrochloric acid $(1 \mathrm{ml})$ was added as a cyclising agent and the reaction mixture was refluxed on water bath at $70-80^{\circ} \mathrm{c}$ gently for about $5-6$ hours. The reaction mixture was then filtered while hot, allowed to cool and neutralised with $\mathrm{NaOH}$. The resulting solid so obtained was filtered, washed several times with distilled water, dried and crystallised from ethanol. M. P. $215^{\circ}$ c., Yield $65 \%$.

Similarly, the remaining substituted pyrimidine - 2 - one derivatives [2(b-j)] were prepared by the same procedure as discussed above.

Physical and Analytical data of compounds [2(a-j)] are presented in Table-2 and Antimicrobial data of compounds $[2(\mathrm{a}-\mathrm{j})]$ are presented in Table-8.

Preparation of 1 - [1"' - (h) - 6"' - (2', - hydroxy phenyl) - pyrimidine - 2'" - thione] - 3 - [1', 2' (dichloro) - dibenz sulphonamide] - 5 - [2,,', 4,", - dichloro phenyl - 2 - pyrazoline derivative [3(a)]

A mixture of 1 - [3"' - (2',' - hydroxy phenyl) - 2', - propene - 1'” - one - 3 - [1', 2' - (dichloro) dibenz sulphonamide] - 5 - [2,", , $4,{ }^{\prime},{ }^{\prime}$ - dichloro phenyl $]-2$ - pyrazoline $[\mathbf{1}(\mathbf{a})](0.001 \mathrm{~mol})$ and thiourea $(0.001 \mathrm{~mol})$ was dissolved in ethanol $(30 \mathrm{ml})$. A $20 \%$ sodium hydroxide solution $(1 \mathrm{ml})$ was added as a cyclising agent and the reaction mixture was refluxed on water bath at $70-80^{\circ} \mathrm{c}$ gently for about 5-6 hours. The reaction mixture was then filtered while hot, allowed to cool and neutralised with dilute $\mathrm{HCl}$. The resulting solid so obtained was filtered, washed several times with distilled water, dried and crystallised from ethanol. M. P. $223^{\circ} \mathrm{c}$., Yield $65 \%$.

Similarly, the remaining substituted pyrimidine - 2 - thione derivatives $[\mathbf{3}(\mathbf{b}-\mathbf{j})]$ were prepared by the same procedure as discussed above.

Physical and Analytical data of compounds [3(a-j)] are presented in Table-3 and Antimicrobial data of compounds [3(a-j)] are presented in Table-9. 
Preparation of 1 - [1'” - (h) - 5', - (2',' - hydroxy phenyl) - 2'” - pyrazolinyl] - 3 - [1', 2' -(dichloro) dibenz sulphonamide] - 5 -[2,', , 4,", - dichloro phenyl] - 2 - pyrazoline derivative [4(a)] [intermediate compound]

To a solution of 1 - [3', - (2',' - hydroxy phenyl) - 2', - propene - 1', - one] - 3 - [1', 2' - (dichloro) dibenz sulphonamide] - 5 - [2,,', , 4,', - dichloro phenyl] - 2 - pyrazoline [1(a)] $(0.001 \mathrm{~mol})$ in ethanol $(30 \mathrm{ml})$, a $99 \%$ hydrazine hydrate $(0.0015 \mathrm{~mol})$ was added drop-wise and the reaction mixture was refluxed on water bath at $70-80^{\circ} \mathrm{c}$ gently for about $4-5$ hours. After the completion of reaction, the reaction mixture was evaporated under vaccum and the resulting solid so obtained was filtered, washed several times with distilled water, dried and crystallised from ethanol. M. P. $162^{\circ}$ c., Yield $68 \%$.

Similarly, the remaining substituted 1 - $(\mathrm{H})$-pyrazoline derivatives [4(b-j)] [Intermediate compounds] were prepared by the same procedure as discussed above.

Physical and Analytical data of compounds [4(a-j)] are presented in Table-4 and Antimicrobial data of compounds $[4(\mathrm{a}-\mathrm{j})]$ are presented in Table-10.

Preparation of 1 - [1", - (p - acetanilide sulphonyl) - 5,' - (2,', - hydroxy phenyl) - 2,' - pyrazolinyl] - 3 - [1', 2' - (dichloro) - dibenz sulphonamide] - 5 - [2,,,', 4,,,' - dichloro phenyl] - 2 - pyrazoline derivative [5(a)]

A solution of 1 - [1' - (H) - 5', - (2',' -hydroxy phenyl) - 2', - pyrazolinyl $]$ - 3 - [1', 2' - (dichloro) dibenz sulphonamide] - 5 - [2,,, , 4,,, - dichloro phenyl] - 2 - pyrazoline [4(a)] [Intermediate compound] $(0.001 \mathrm{~mol})$ in pyridine $(30 \mathrm{ml})$ was cooled in an ice-bath at $0-5^{\circ} \mathrm{c}$ and to it, $\mathrm{p}$ - acetanilide sulphonyl chloride $(0.001 \mathrm{~mol})$ was added slowly with constant stirring. The reaction mixture was further stirred for 4-5 hours and the content was then poured over crushed ice and acidified with dilute $\mathrm{HCl}$. The resulting solid so obtained was filtered, washed several times with distilled water, dried and crystallised from ethanol. M. P. $205^{\circ}$ c., Yield $66 \%$.

Similarly, the remaining substituted $\mathrm{p}$ - acetanilide sulphonyl derivatives $[\mathbf{5}(\mathbf{b}-\mathbf{j})]$ were prepared by the same precedure as discussed above.

Physical and Analytical data of compounds [5(a-j)] are presented in Table-5 and Antimicrobial data of compounds $[5(\mathrm{a}-\mathrm{j})]$ are presented in Table-11.

Preparation of 1 - [1'” - (benzoyl) - 5' - (2',' - hydroxy phenyl) - 2'” - pyrazolinyl] - 3 - [1', 2' - (dichloro) dibenz sulphonamide] - 5- [2,", , 4,',' - dichloro phenyl] - 2 - pyrazoline derivative [6(a)]

To a solution of 1 - [1', - (H) - 5', - (2,', -hydroxy phenyl) - 2', - pyrazolinyl] - 3 - [1', 2' - (dichloro) dibenz sulphonamide] - 5 - [2,,, , 4,,, - dichloro phenyl] - 2 - pyrazoline [4(a)] [Intermediate compound] $(0.001 \mathrm{~mol})$ in pyridine $(30 \mathrm{ml})$, benzoyl chloride $(0.001 \mathrm{~mol})$ was added drop-wise with constant stirring and the reaction mixture was further stirred at room temperature for 4-5 hours. The content was then poured over crushed ice and ice and acidified with dilute $\mathrm{HCl}$. The resulting solid so obtained was filtered, washed several times with distilled water, dried and crystallised from ethanol. M. P. $186^{\circ}$ c., Yield $65 \%$.

Similarly, the remaining substituted benzoyl derivatives $[\mathbf{6}(\mathbf{b}-\mathbf{j})]$ were prepared by the same procedure as discussed above.

Physical and Analytical data of compounds [6(a-j)] are presented in Table-6 and Antimicrobial data of compounds $[6(\mathrm{a}-\mathrm{j})]$ are presented in Table-12. 
Table 1. PHYSICAL AND ANALYTICAL DATA OF COMPOUNDS [1(a-j)]

\begin{tabular}{|c|c|c|c|c|c|c|c|}
\hline \multirow{2}{*}{$\begin{array}{l}\text { Compd. } \\
\text { No. }\end{array}$} & \multirow[t]{2}{*}{$\mathrm{R}$} & \multirow{2}{*}{$\begin{array}{c}\text { M. F. } \\
{[\text { M. W. g/m)] }}\end{array}$} & \multirow{2}{*}{$\begin{array}{l}\text { M. } \\
\text { P. } \\
(\mathrm{oC})\end{array}$} & \multirow{2}{*}{$\begin{array}{l}\text { Yield } \\
(\%)\end{array}$} & \multicolumn{2}{|c|}{$\begin{array}{l}\% \text { Analysis } \\
\quad \text { (Calcd.) }\end{array}$} & Found \\
\hline & & & & & $\mathrm{C} \%$ & $\mathrm{H} \%$ & $\mathrm{~N} \%$ \\
\hline 1 a. & $2-\mathrm{OH}$ & $\begin{array}{c}\mathrm{C}_{30} \mathrm{H}_{21} \mathrm{~N}_{3} \mathrm{O}_{4} \mathrm{SCI}_{4} \\
(661.0)\end{array}$ & 152 & 70 & $\begin{array}{c}54.43 \\
(54.46)\end{array}$ & $\begin{array}{c}3.16 \\
(3.18)\end{array}$ & $\begin{array}{c}6.34 \\
(6.35)\end{array}$ \\
\hline $1 \mathrm{~b}$. & 2-CI & $\begin{array}{c}\mathrm{C}_{30} \mathrm{H}_{20} \mathrm{~N}_{3} \mathrm{O}_{3} \mathrm{SCI}_{5} \\
(679.5)\end{array}$ & 164 & 65 & $\begin{array}{c}52.96 \\
(52.98)\end{array}$ & $\begin{array}{c}2.90 \\
(2.94)\end{array}$ & $\begin{array}{c}6.15 \\
(6.18)\end{array}$ \\
\hline $1 \mathrm{c}$. & 3-CI & $\begin{array}{c}\mathrm{C}_{30} \mathrm{H}_{20} \mathrm{~N}_{3} \mathrm{O}_{3} \mathrm{SCI}_{5} \\
(679.5)\end{array}$ & 168 & 68 & $\begin{array}{c}52.96 \\
(52.98)\end{array}$ & $\begin{array}{l}2.91 \\
(2.94)\end{array}$ & $\begin{array}{c}6.15 \\
(6.18)\end{array}$ \\
\hline $1 \mathrm{~d}$ & $2-\mathrm{NO}_{2}$ & $\begin{array}{c}\mathrm{C}_{30} \mathrm{H}_{20} \mathrm{~N}_{4} \mathrm{O}_{5} \mathrm{SCI}_{4} \\
(690.0)\end{array}$ & 160 & 64 & $\begin{array}{c}52.13 \\
(52.17)\end{array}$ & $\begin{array}{c}2.88 \\
(2.90)\end{array}$ & $\begin{array}{c}8.08 \\
(8.11)\end{array}$ \\
\hline $1 \mathrm{e}$. & $3-\mathrm{NO}_{2}$ & $\begin{array}{c}\mathrm{C}_{30} \mathrm{H}_{20} \mathrm{~N}_{4} \mathrm{O}_{5} \mathrm{SCI}_{4} \\
(690.0)\end{array}$ & 174 & 62 & $\begin{array}{c}52.14 \\
(52.17)\end{array}$ & $\begin{array}{c}2.88 \\
(2.90)\end{array}$ & $\begin{array}{c}8.07 \\
(8.11)\end{array}$ \\
\hline $1 \mathrm{f}$. & $4-\mathrm{NO}_{2}$ & $\begin{array}{c}\mathrm{C}_{30} \mathrm{H}_{20} \mathrm{~N}_{4} \mathrm{O}_{5} \mathrm{SCI}_{4} \\
(690.0)\end{array}$ & 165 & 60 & $\begin{array}{c}52.13 \\
(52.17)\end{array}$ & $\begin{array}{c}2.87 \\
(2.90)\end{array}$ & $\begin{array}{c}8.07 \\
(8.11)\end{array}$ \\
\hline $1 \mathrm{~g}$. & $2-\mathrm{OCH}_{3}$ & $\begin{array}{c}\mathrm{C}_{31} \mathrm{H}_{23} \mathrm{~N}_{3} \mathrm{O}_{4} \mathrm{SCI}_{4} \\
(675.0)\end{array}$ & 148 & 67 & $\begin{array}{l}55.09 \\
(55.11)\end{array}$ & $\begin{array}{c}3.37 \\
(3.41)\end{array}$ & $\begin{array}{c}6.19 \\
(6.22)\end{array}$ \\
\hline $1 \mathrm{~h}$. & $3-\mathrm{OCH}_{3}-4-\mathrm{OH}$ & $\begin{array}{c}\mathrm{C}_{31} \mathrm{H}_{23} \mathrm{~N}_{3} \mathrm{O}_{5} \mathrm{SCI}_{4} \\
(691.0)\end{array}$ & 163 & 65 & $\begin{array}{c}53.82 \\
(53.83)\end{array}$ & $\begin{array}{c}3.30 \\
(3.33)\end{array}$ & $\begin{array}{c}6.06 \\
(6.08)\end{array}$ \\
\hline $1 \mathrm{i}$. & $4-\mathrm{OCH}_{3}$ & $\begin{array}{c}\mathrm{C}_{31} \mathrm{H}_{23} \mathrm{~N}_{3} \mathrm{O}_{4} \mathrm{SCI}_{4} \\
(675.0)\end{array}$ & 155 & 68 & $\begin{array}{c}55.10 \\
(55.11)\end{array}$ & $\begin{array}{c}3.38 \\
(3.41)\end{array}$ & $\begin{array}{c}6.19 \\
(6.22)\end{array}$ \\
\hline $1 \mathrm{j}$. & $\left.3,4,5-\mathrm{OCH}_{3}\right)_{3}$ & $\begin{array}{c}\mathrm{C}_{33} \mathrm{H}_{27} \mathrm{~N}_{3} \mathrm{O}_{6} \mathrm{SCI}_{4} \\
(735.0)\end{array}$ & 166 & 64 & $\begin{array}{c}53.85 \\
(53.88) \\
\end{array}$ & $\begin{array}{c}3.65 \\
(3.67)\end{array}$ & $\begin{array}{r}5.69 \\
(5.71) \\
\end{array}$ \\
\hline
\end{tabular}

Table 2. PHYSICAL AND ANALYTICAL DATA OF COMPOUNDS [2(a-j)]

\begin{tabular}{|c|c|c|c|c|c|c|c|}
\hline \multirow{2}{*}{$\begin{array}{l}\text { Compd. } \\
\text { No. }\end{array}$} & \multirow{2}{*}{$\mathrm{R}$} & \multirow{2}{*}{$\begin{array}{l}\text { M. F. } \\
\text { W. }(g / m)]\end{array}$} & \multirow{2}{*}{$\begin{array}{l}\text { M. P. } \\
\left({ }^{\circ} \mathrm{C}\right)\end{array}$} & \multirow{2}{*}{$\begin{array}{l}\text { Yield } \\
(\%)\end{array}$} & $\%$ Analysis & \multicolumn{2}{|c|}{ Found (Calcd.) } \\
\hline & & & & & $\mathrm{C} \%$ & $\mathrm{H} \%$ & $\mathrm{~N} \%$ \\
\hline 2 a. & $2-\mathrm{OH}$ & $\begin{array}{c}\mathrm{C}_{31} \mathrm{H}_{23} \mathrm{~N}_{5} \mathrm{O}_{4} \mathrm{SCI}_{4} \\
(703.0)\end{array}$ & 215 & 65 & $\begin{array}{c}52.90 \\
(52.92)\end{array}$ & $\begin{array}{c}3.24 \\
(3.27)\end{array}$ & $\begin{array}{c}9.92 \\
(9.96)\end{array}$ \\
\hline $2 \mathrm{~b}$. & 2-CI & $\begin{array}{c}\mathrm{C}_{31} \mathrm{H}_{22} \mathrm{~N}_{5} \mathrm{O}_{3} \mathrm{SCI}_{5} \\
(721.5)\end{array}$ & 187 & 62 & $\begin{array}{c}51.53 \\
(51.56)\end{array}$ & $\begin{array}{c}3.01 \\
(3.05)\end{array}$ & $\begin{array}{l}9.67 \\
(9.70)\end{array}$ \\
\hline $2 \mathrm{c}$. & $3-\mathrm{CI}$ & $\begin{array}{c}\mathrm{C}_{31} \mathrm{H}_{22} \mathrm{~N}_{5} \mathrm{O}_{3} \mathrm{SCI}_{5} \\
(721.5)\end{array}$ & 192 & 68 & $\begin{array}{c}51.53 \\
(51.56)\end{array}$ & $\begin{array}{l}3.02 \\
(3.05)\end{array}$ & $\begin{array}{c}9.68 \\
(9.70)\end{array}$ \\
\hline $2 \mathrm{~d}$. & $2-\mathrm{NO}_{2}$ & $\begin{array}{c}\mathrm{C}_{31} \mathrm{H}_{22} \mathrm{~N}_{6} \mathrm{O}_{5} \mathrm{SCI}_{4} \\
(732.0)\end{array}$ & 178 & 65 & $\begin{array}{c}50.78 \\
(50.82)\end{array}$ & $\begin{array}{l}2.97 \\
(3.00)\end{array}$ & $\begin{array}{c}11.45 \\
(11.47)\end{array}$ \\
\hline 2 e. & $3-\mathrm{NO}_{2}$ & $\begin{array}{c}\mathrm{C}_{31} \mathrm{H}_{22} \mathrm{~N}_{6} \mathrm{O}_{5} \mathrm{SCI}_{4} \\
(732.0)\end{array}$ & 185 & 64 & $\begin{array}{c}50.79 \\
(50.82)\end{array}$ & $\begin{array}{l}2.97 \\
(3.00)\end{array}$ & $\begin{array}{l}11.44 \\
(11.47)\end{array}$ \\
\hline $2 \mathrm{f}$. & $4-\mathrm{NO}_{2}$ & $\begin{array}{c}\mathrm{C}_{31} \mathrm{H}_{22} \mathrm{~N}_{6} \mathrm{O}_{5} \mathrm{SCI}_{4} \\
(732.0)\end{array}$ & 172 & 60 & $\begin{array}{c}50.78 \\
(50.82)\end{array}$ & $\begin{array}{l}2.96 \\
(3.00)\end{array}$ & $\begin{array}{l}11.44 \\
(11.47)\end{array}$ \\
\hline $2 \mathrm{~g}$. & $2-\mathrm{OCH}_{3}$ & $\begin{array}{c}\mathrm{C}_{32} \mathrm{H}_{25} \mathrm{~N}_{5} \mathrm{O}_{4} \mathrm{SCI}_{4} \\
(717.0)\end{array}$ & 203 & 67 & $\begin{array}{c}53.55 \\
(53.56)\end{array}$ & $\begin{array}{l}3.46 \\
(3.49)\end{array}$ & $\begin{array}{c}9.72 \\
(9.76)\end{array}$ \\
\hline $2 \mathrm{~h}$. & $\begin{array}{c}3-\mathrm{OCH}_{3}-4- \\
\mathrm{OH}\end{array}$ & $\begin{array}{c}\mathrm{C}_{32} \mathrm{H}_{25} \mathrm{~N}_{5} \mathrm{O}_{5} \mathrm{SCI}_{4} \\
(733.0)\end{array}$ & 175 & 66 & $\begin{array}{c}52.36 \\
(52.39)\end{array}$ & $\begin{array}{l}3.38 \\
(3.41)\end{array}$ & $\begin{array}{c}9.53 \\
(9.55)\end{array}$ \\
\hline $2 \mathrm{i}$. & $4-\mathrm{OCH}_{3}$ & $\begin{array}{c}\mathrm{C}_{32} \mathrm{H}_{25} \mathrm{~N}_{5} \mathrm{O}_{4} \mathrm{SCI}_{4} \\
(717.0)\end{array}$ & 195 & 62 & $\begin{array}{c}53.54 \\
(53.56)\end{array}$ & $\begin{array}{l}3.46 \\
(3.49)\end{array}$ & $\begin{array}{l}9.73 \\
(9.76)\end{array}$ \\
\hline $2 \mathrm{j}$. & $\begin{array}{l}3,4,5- \\
\left(\mathrm{OCH}_{3}\right)_{3}\end{array}$ & $\begin{array}{c}\mathrm{C}_{34} \mathrm{H}_{29} \mathrm{~N}_{5} \mathrm{O}_{6} \mathrm{SCI}_{4} \\
(777.0)\end{array}$ & 197 & 64 & $\begin{array}{c}52.49 \\
(52.51)\end{array}$ & $\begin{array}{c}3.71 \\
(3.73)\end{array}$ & $\begin{array}{c}8.97 \\
(9.00) \\
\end{array}$ \\
\hline
\end{tabular}


Table 3. PHYSICAL AND ANALYTICAL DATA OF COMPOUNDS [3(a-j)]

\begin{tabular}{|c|c|c|c|c|c|c|c|}
\hline \multirow{2}{*}{$\begin{array}{l}\text { Comp. } \\
\text { No. }\end{array}$} & \multirow[t]{2}{*}{$\mathrm{R}$} & \multirow{2}{*}{$\begin{array}{c}\text { M. F. } \\
{[\text { M. W. }(\mathrm{g} / \mathrm{m})]}\end{array}$} & \multirow{2}{*}{$\begin{array}{l}\text { M. P. } \\
(\mathrm{oC})\end{array}$} & \multirow{2}{*}{$\begin{array}{l}\text { Yield } \\
(\%)\end{array}$} & \multicolumn{2}{|c|}{$\begin{array}{l}\% \text { Analysis } \\
\text { (Calcd.) }\end{array}$} & Found \\
\hline & & & & & $\mathrm{C} \%$ & $\mathrm{H} \%$ & $\mathrm{~N} \%$ \\
\hline 3 a. & $2-\mathrm{OH}$ & $\begin{array}{c}\mathrm{C}_{31} \mathrm{H}_{23} \mathrm{~N}_{5} \mathrm{O}_{3} \mathrm{~S}_{2} \mathrm{CI}_{4} \\
(719.0)\end{array}$ & 223 & 65 & $\begin{array}{c}51.71 \\
(51.74)\end{array}$ & $\begin{array}{c}3.18 \\
(3.20)\end{array}$ & $\begin{array}{c}9.70 \\
(9.73)\end{array}$ \\
\hline 3 b. & 2-CI & $\begin{array}{c}\mathrm{C}_{31} \mathrm{H}_{22} \mathrm{~N}_{5} \mathrm{O}_{2} \mathrm{~S}_{2} \mathrm{Cl}_{5} \\
(735.5)\end{array}$ & 195 & 56 & $\begin{array}{c}50.42 \\
(50.44)\end{array}$ & $\begin{array}{l}2.95 \\
(2.98)\end{array}$ & $\begin{array}{l}9.45 \\
(9.49)\end{array}$ \\
\hline $3 \mathrm{c}$. & 3-CI & $\begin{array}{c}\mathrm{C}_{31} \mathrm{H}_{22} \mathrm{~N}_{5} \mathrm{O}_{2} \mathrm{~S}_{2} \mathrm{Cl}_{5} \\
(737.5)\end{array}$ & 202 & 64 & $\begin{array}{c}50.41 \\
(50.44)\end{array}$ & $\begin{array}{l}2.95 \\
(2.98)\end{array}$ & $\begin{array}{l}9.46 \\
(9.49)\end{array}$ \\
\hline $3 \mathrm{~d}$. & $2-\mathrm{NO}_{2}$ & $\begin{array}{c}\mathrm{C}_{31} \mathrm{H}_{22} \mathrm{~N}_{6} \mathrm{O}_{4} \mathrm{~S}_{2} \mathrm{Cl}_{4} \\
(748.0)\end{array}$ & 185 & 67 & $\begin{array}{c}49.69 \\
(49.73)\end{array}$ & $\begin{array}{l}2.91 \\
(2.94)\end{array}$ & $\begin{array}{r}11.20 \\
(11.23)\end{array}$ \\
\hline 3 e. & $3-\mathrm{NO}_{2}$ & $\begin{array}{c}\mathrm{C}_{31} \mathrm{H}_{22} \mathrm{~N}_{6} \mathrm{O}_{4} \mathrm{~S}_{2} \mathrm{Cl}_{4} \\
(748.0)\end{array}$ & 190 & 59 & $\begin{array}{c}49.70 \\
(49.73)\end{array}$ & $\begin{array}{l}2.92 \\
(2.94)\end{array}$ & $\begin{array}{r}11.20 \\
(11.23)\end{array}$ \\
\hline $3 \mathrm{f}$. & $4-\mathrm{NO}_{2}$ & $\begin{array}{c}\mathrm{C}_{31} \mathrm{H}_{22} \mathrm{~N}_{6} \mathrm{O}_{4} \mathrm{~S}_{2} \mathrm{Cl}_{4} \\
(748.0)\end{array}$ & 182 & 58 & $\begin{array}{c}49.70 \\
(49.73)\end{array}$ & $\begin{array}{l}2.91 \\
(2.94)\end{array}$ & $\begin{array}{r}11.19 \\
(11.23)\end{array}$ \\
\hline $3 \mathrm{~g}$. & $2-\mathrm{OCH}_{3}$ & $\begin{array}{c}\mathrm{C}_{32} \mathrm{H}_{25} \mathrm{~N}_{5} \mathrm{O}_{3} \mathrm{~S}_{2} \mathrm{Cl}_{4} \\
(733.0)\end{array}$ & 217 & 67 & $\begin{array}{c}52.37 \\
(52.39)\end{array}$ & $\begin{array}{c}3.38 \\
(3.41)\end{array}$ & $\begin{array}{c}9.52 \\
(9.55)\end{array}$ \\
\hline $3 \mathrm{~h}$. & $\begin{array}{c}3-\mathrm{OCH}_{3}^{-} \\
4-\mathrm{OH}\end{array}$ & $\begin{array}{c}\mathrm{C}_{32} \mathrm{H}_{25} \mathrm{~N}_{5} \mathrm{O}_{4} \mathrm{~S}_{2} \mathrm{Cl}_{4} \\
(749.0)\end{array}$ & 180 & 68 & $\begin{array}{c}51.23 \\
(51.27)\end{array}$ & $\begin{array}{c}3.33 \\
(3.34)\end{array}$ & $\begin{array}{c}9.30 \\
(9.34)\end{array}$ \\
\hline 3 i. & $4-\mathrm{OCH}_{3}$ & $\begin{array}{c}\mathrm{C}_{32} \mathrm{H}_{25} \mathrm{~N}_{5} \mathrm{O}_{3} \mathrm{~S}_{2} \mathrm{Cl}_{4} \\
(733.0)\end{array}$ & 212 & 62 & $\begin{array}{c}52.36 \\
(52.39)\end{array}$ & $\begin{array}{c}3.38 \\
(3.41)\end{array}$ & $\begin{array}{c}9.53 \\
(9.55)\end{array}$ \\
\hline $3 \mathrm{j}$. & $\begin{array}{l}3,4,5- \\
\left(\mathrm{OCH}_{3}\right)_{3}\end{array}$ & $\begin{array}{c}\mathrm{C}_{34} \mathrm{H}_{29} \mathrm{~N}_{5} \mathrm{O}_{5} \mathrm{~S}_{2} \mathrm{Cl}_{4} \\
(793.0)\end{array}$ & 207 & 66 & $\begin{array}{c}51.42 \\
(51.45) \\
\end{array}$ & $\begin{array}{c}3.64 \\
(3.66)\end{array}$ & $\begin{array}{r}8.81 \\
(8.33) \\
\end{array}$ \\
\hline
\end{tabular}

Table 4. PHYSICAL AND ANALYTICAL DATA OF COMPOUNDS [4(a-j)]

\begin{tabular}{|c|c|c|c|c|c|c|c|}
\hline \multirow{2}{*}{$\begin{array}{l}\text { Compd. } \\
\text { No. }\end{array}$} & \multirow{2}{*}{$\mathrm{R}$} & \multirow{2}{*}{$\begin{array}{c}\text { M. F. } \\
{[\mathrm{M} . \mathrm{W} .(\mathrm{g} / \mathrm{m})]}\end{array}$} & \multirow{2}{*}{$\begin{array}{l}\text { M. P. } \\
\left({ }^{\circ} \mathrm{C}\right)\end{array}$} & \multirow{2}{*}{$\begin{array}{l}\text { Yield } \\
(\%)\end{array}$} & \multicolumn{2}{|c|}{$\begin{array}{l}\text { \% Analysis } \\
\text { (Calcd.) }\end{array}$} & Found \\
\hline & & & & & $\mathrm{C} \%$ & $\mathrm{H} \%$ & $\mathrm{~N} \%$ \\
\hline 4 a. & $2-\mathrm{OH}$ & $\begin{array}{c}\mathrm{C}_{30} \mathrm{H}_{21} \mathrm{~N}_{5} \mathrm{O}_{3} \mathrm{SCl}_{4} \\
(673.0)\end{array}$ & 162 & 68 & $\begin{array}{c}53.46 \\
(53.49)\end{array}$ & $\begin{array}{c}3.10 \\
(3.12)\end{array}$ & $\begin{array}{c}10.37 \\
(10.40)\end{array}$ \\
\hline $4 \mathrm{~b}$ & 2-CI & $\begin{array}{c}\mathrm{C}_{30} \mathrm{H}_{20} \mathrm{~N}_{5} \mathrm{O}_{2} \mathrm{SCl}_{5} \\
(691.5)\end{array}$ & 167 & 64 & $\begin{array}{c}52.02 \\
(52.06)\end{array}$ & $\begin{array}{l}2.86 \\
(2.89)\end{array}$ & $\begin{array}{c}10.09 \\
(10.12)\end{array}$ \\
\hline $4 \mathrm{c}$. & 3-CI & $\begin{array}{c}\mathrm{C}_{30} \mathrm{H}_{20} \mathrm{~N}_{5} \mathrm{O}_{2} \mathrm{SCl}_{5} \\
(691.5)\end{array}$ & 172 & 65 & $\begin{array}{c}52.03 \\
(52.06)\end{array}$ & $\begin{array}{l}2.85 \\
(2.89)\end{array}$ & $\begin{array}{c}10.09 \\
(10.12)\end{array}$ \\
\hline $4 \mathrm{~d}$ & $2-\mathrm{NO}_{2}$ & $\begin{array}{c}\mathrm{C}_{30} \mathrm{H}_{20} \mathrm{~N}_{6} \mathrm{O}_{4} \mathrm{SCl}_{4} \\
(702.0)\end{array}$ & 158 & 68 & $\begin{array}{c}51.25 \\
(51.28)\end{array}$ & $\begin{array}{l}2.83 \\
(2.85)\end{array}$ & $\begin{array}{l}11.94 \\
(11.96)\end{array}$ \\
\hline $4 \mathrm{e}$. & $3-\mathrm{NO}_{2}$ & $\begin{array}{c}\mathrm{C}_{30} \mathrm{H}_{20} \mathrm{~N}_{6} \mathrm{O}_{4} \mathrm{SCl}_{4} \\
(702.0)\end{array}$ & 165 & 63 & $\begin{array}{l}51.25 \\
(51.28)\end{array}$ & $\begin{array}{l}2.82 \\
(2.85)\end{array}$ & $\begin{array}{c}11.95 \\
(11.96)\end{array}$ \\
\hline $4 \mathrm{f}$. & $4-\mathrm{NO}_{2}$ & $\begin{array}{c}\mathrm{C}_{30} \mathrm{H}_{20} \mathrm{~N}_{6} \mathrm{O}_{4} \mathrm{SCl}_{4} \\
(702.0)\end{array}$ & 155 & 62 & $\begin{array}{c}51.26 \\
(51.28)\end{array}$ & $\begin{array}{l}2.83 \\
(2.85)\end{array}$ & $\begin{array}{l}11.94 \\
(11.96)\end{array}$ \\
\hline $4 \mathrm{~g}$. & $2-\mathrm{OCH}_{3}$ & $\begin{array}{c}\mathrm{C}_{31} \mathrm{H}_{23} \mathrm{~N}_{5} \mathrm{O}_{3} \mathrm{SCl}_{4} \\
(687.0)\end{array}$ & 160 & 66 & $\begin{array}{c}54.11 \\
(54.15)\end{array}$ & $\begin{array}{l}3.31 \\
(3.35)\end{array}$ & $\begin{array}{l}10.16 \\
(10.19)\end{array}$ \\
\hline $4 \mathrm{~h}$. & $\begin{array}{c}3-\mathrm{OCH}_{3}- \\
4-\mathrm{OH}\end{array}$ & $\begin{array}{c}\mathrm{C}_{31} \mathrm{H}_{23} \mathrm{~N}_{5} \mathrm{O}_{4} \mathrm{SCl}_{4} \\
(703.0)\end{array}$ & 168 & 70 & $\begin{array}{c}52.90 \\
(52.92)\end{array}$ & $\begin{array}{l}3.24 \\
(3.27)\end{array}$ & $\begin{array}{c}9.92 \\
(9.96)\end{array}$ \\
\hline $4 \mathrm{i}$. & $4-\mathrm{OCH}_{3}$ & $\begin{array}{c}\mathrm{C}_{31} \mathrm{H}_{23} \mathrm{~N}_{5} \mathrm{O}_{3} \mathrm{SCl}_{4} \\
(687.0)\end{array}$ & 162 & 63 & $\begin{array}{c}54.13 \\
(54.15)\end{array}$ & $\begin{array}{l}3.32 \\
(3.35)\end{array}$ & $\begin{array}{c}10.17 \\
(10.19)\end{array}$ \\
\hline $4 \mathrm{j}$. & $\begin{array}{l}3,4,5- \\
\left(\mathrm{OCH}_{3}\right)_{3}\end{array}$ & $\begin{array}{c}\mathrm{C}_{33} \mathrm{H}_{27} \mathrm{~N}_{5} \mathrm{O}_{5} \mathrm{SCl}_{4} \\
(747.0)\end{array}$ & 178 & 65 & $\begin{array}{c}52.98 \\
(53.01)\end{array}$ & $\begin{array}{c}3.59 \\
(3.61)\end{array}$ & $\begin{array}{c}9.34 \\
(9.37)\end{array}$ \\
\hline
\end{tabular}


Table 5. PHYSICAL AND ANALYTICAL DATA OF COMPOUNDS [5(a-j)]

\begin{tabular}{|c|c|c|c|c|c|c|c|}
\hline \multirow{2}{*}{$\begin{array}{l}\text { Comp. } \\
\text { No. }\end{array}$} & \multirow[t]{2}{*}{$\mathrm{R}$} & \multirow{2}{*}{$\begin{array}{l}\text { M. F. } \\
\text { [M. W. }(\mathrm{g} / \mathrm{m})]\end{array}$} & \multirow{2}{*}{$\begin{array}{l}\text { M. P. } \\
\text { (oC) }\end{array}$} & \multirow{2}{*}{$\begin{array}{l}\text { Yield } \\
(\%)\end{array}$} & \multicolumn{3}{|c|}{$\begin{array}{c}\% \text { Analysis } \\
\text { Found (Calcd.) }\end{array}$} \\
\hline & & & & & C\% & H\% & $\mathrm{N} \%$ \\
\hline 5 a. & $2-\mathrm{OH}$ & $\begin{array}{c}\mathrm{C}_{38} \mathrm{H}_{30} \mathrm{~N}_{6} \mathrm{O}_{6} \mathrm{~S}_{2} \mathrm{Cl}_{4} \\
(872.0)\end{array}$ & 205 & 66 & $\begin{array}{c}52.27 \\
(52.29)\end{array}$ & $\begin{array}{c}3.41 \\
(3.44)\end{array}$ & $\begin{array}{c}9.60 \\
(9.63)\end{array}$ \\
\hline $5 \mathrm{~b}$ & $2-\mathrm{CI}$ & $\begin{array}{c}\mathrm{C}_{38} \mathrm{H}_{29} \mathrm{~N}_{6} \mathrm{O}_{5} \mathrm{~S}_{2} \mathrm{Cl}_{5} \\
(890.5)\end{array}$ & 183 & 68 & $\begin{array}{c}51.18 \\
(51.21)\end{array}$ & $\begin{array}{c}3.24 \\
(3.26)\end{array}$ & $\begin{array}{c}9.40 \\
(9.43)\end{array}$ \\
\hline $5 \mathrm{c}$. & $3-\mathrm{CI}$ & $\begin{array}{c}\mathrm{C}_{38} \mathrm{H}_{29} \mathrm{~N}_{6} \mathrm{O}_{5} \mathrm{~S}_{2} \mathrm{Cl}_{5} \\
(890.5)\end{array}$ & 175 & 65 & $\begin{array}{c}51.18 \\
(51.21)\end{array}$ & $\begin{array}{c}3.23 \\
(3.26)\end{array}$ & $\begin{array}{c}9.41 \\
(9.43)\end{array}$ \\
\hline $5 \mathrm{~d}$. & $2-\mathrm{NO}_{2}$ & $\begin{array}{c}\mathrm{C}_{38} \mathrm{H}_{29} \mathrm{~N}_{7} \mathrm{O}_{7} \mathrm{~S}_{2} \mathrm{Cl}_{4} \\
(901.0)\end{array}$ & 180 & 64 & $\begin{array}{c}50.57 \\
(50.61)\end{array}$ & $\begin{array}{c}3.20 \\
(3.22)\end{array}$ & $\begin{array}{c}10.85 \\
(10.88)\end{array}$ \\
\hline 5 e. & $3-\mathrm{NO}_{2}$ & $\begin{array}{c}\mathrm{C}_{38} \mathrm{H}_{29} \mathrm{~N}_{7} \mathrm{O}_{7} \mathrm{~S}_{2} \mathrm{Cl}_{4} \\
(901.0)\end{array}$ & 185 & 62 & $\begin{array}{c}50.58 \\
(50.61)\end{array}$ & $\begin{array}{c}3.19 \\
(3.22)\end{array}$ & $\begin{array}{c}10.86 \\
(10.88)\end{array}$ \\
\hline $5 \mathrm{f}$. & $4-\mathrm{NO}_{2}$ & $\begin{array}{c}\mathrm{C}_{38} \mathrm{H}_{29} \mathrm{~N}_{7} \mathrm{O}_{7} \mathrm{~S}_{2} \mathrm{Cl}_{4} \\
(901.0)\end{array}$ & 177 & 60 & $\begin{array}{c}50.58 \\
(50.61)\end{array}$ & $\begin{array}{c}3.20 \\
(3.22)\end{array}$ & $\begin{array}{c}10.85 \\
(10.88)\end{array}$ \\
\hline $5 \mathrm{~g}$. & $2-\mathrm{OCH}_{3}$ & $\begin{array}{c}\mathrm{C}_{39} \mathrm{H}_{32} \mathrm{~N}_{6} \mathrm{O}_{6} \mathrm{~S}_{2} \mathrm{Cl}_{4} \\
(886.0)\end{array}$ & 153 & 66 & $\begin{array}{c}52.80 \\
(52.82)\end{array}$ & $\begin{array}{c}3.57 \\
(3.61)\end{array}$ & $\begin{array}{l}9.46 \\
(9.48)\end{array}$ \\
\hline $5 \mathrm{~h}$. & $\begin{array}{c}3-\mathrm{OCH}_{3}- \\
4-\mathrm{OH}\end{array}$ & $\begin{array}{c}\mathrm{C}_{39} \mathrm{H}_{32} \mathrm{~N}_{6} \mathrm{O}_{7} \mathrm{~S}_{2} \mathrm{Cl}_{4} \\
(902.0)\end{array}$ & 168 & 65 & $\begin{array}{c}51.85 \\
(51.88)\end{array}$ & $\begin{array}{c}3.52 \\
(3.55)\end{array}$ & $\begin{array}{c}9.27 \\
(9.31)\end{array}$ \\
\hline $5 \mathrm{i}$. & $4-\mathrm{OCH}_{3}$ & $\begin{array}{c}\mathrm{C}_{39} \mathrm{H}_{32} \mathrm{~N}_{6} \mathrm{O}_{6} \mathrm{~S}_{2} \mathrm{Cl}_{4} \\
(886.0)\end{array}$ & 158 & 63 & $\begin{array}{c}52.79 \\
(52.82)\end{array}$ & $\begin{array}{c}3.58 \\
(3.61)\end{array}$ & $\begin{array}{c}9.45 \\
(9.48)\end{array}$ \\
\hline $5 \mathrm{j}$. & $\begin{array}{l}3,4,5- \\
\left(\mathrm{OCH}_{3}\right)_{3}\end{array}$ & $\begin{array}{c}\mathrm{C}_{41} \mathrm{H}_{36} \mathrm{~N}_{6} \mathrm{O}_{8} \mathrm{~S}_{2} \mathrm{Cl}_{4} \\
(946.0)\end{array}$ & 196 & 67 & $\begin{array}{c}51.97 \\
(52.00)\end{array}$ & $\begin{array}{c}3.78 \\
(3.80)\end{array}$ & $\begin{array}{c}8.86 \\
(8.88)\end{array}$ \\
\hline
\end{tabular}

Table 6. PHYSICAL AND ANALYTICAL DATA OF COMPOUNDS [6(a-j)]

\begin{tabular}{|c|c|c|c|c|c|c|c|}
\hline \multirow{2}{*}{$\begin{array}{l}\text { Comp. } \\
\text { No. }\end{array}$} & \multirow[t]{2}{*}{$\mathrm{R}$} & \multirow{2}{*}{$\begin{array}{c}\text { M. F. } \\
\text { [M. W. (g/m)] }\end{array}$} & \multirow{2}{*}{$\begin{array}{l}\text { M. P. } \\
(\mathrm{oC})\end{array}$} & \multirow{2}{*}{$\begin{array}{l}\text { Yield } \\
(\%)\end{array}$} & \multicolumn{3}{|c|}{$\begin{array}{l}\% \text { Analysis } \\
\text { Found (Calcd.) }\end{array}$} \\
\hline & & & & & C\% & $\mathrm{H} \%$ & $\mathrm{~N} \%$ \\
\hline 6 a. & $2-\mathrm{OH}$ & $\begin{array}{c}\mathrm{C}_{37} \mathrm{H}_{27} \mathrm{~N}_{5} \mathrm{O}_{4} \mathrm{SCl}_{4} \\
(779.0)\end{array}$ & 186 & 65 & $\begin{array}{c}56.98 \\
(57.00)\end{array}$ & $\begin{array}{c}3.43 \\
(3.46)\end{array}$ & $\begin{array}{c}8.96 \\
(8.98)\end{array}$ \\
\hline $6 \mathrm{~b}$. & 2-CI & $\begin{array}{c}\mathrm{C}_{37} \mathrm{H}_{26} \mathrm{~N}_{5} \mathrm{O}_{3} \mathrm{SCl}_{5} \\
(797.5)\end{array}$ & 177 & 67 & $\begin{array}{c}55.64 \\
(55.67)\end{array}$ & $\begin{array}{l}3.25 \\
(3.26)\end{array}$ & $\begin{array}{c}8.74 \\
(8.78)\end{array}$ \\
\hline $6 \mathrm{c}$. & 3-CI & $\begin{array}{c}\mathrm{C}_{37} \mathrm{H}_{26} \mathrm{~N}_{5} \mathrm{O}_{3} \mathrm{SCl}_{5} \\
(797.5)\end{array}$ & 170 & 70 & $\begin{array}{c}55.64 \\
(55.67)\end{array}$ & $\begin{array}{c}3.24 \\
(3.26)\end{array}$ & $\begin{array}{c}8.75 \\
(8.78)\end{array}$ \\
\hline $6 \mathrm{~d}$. & $2-\mathrm{NO}_{2}$ & $\begin{array}{c}\mathrm{C}_{37} \mathrm{H}_{26} \mathrm{~N}_{6} \mathrm{O}_{5} \mathrm{SCl}_{4} \\
(808.0)\end{array}$ & 173 & 68 & $\begin{array}{c}54.91 \\
(54.95)\end{array}$ & $\begin{array}{c}3.19 \\
(3.22)\end{array}$ & $\begin{array}{c}10.37 \\
(10.40)\end{array}$ \\
\hline $6 \mathrm{e}$. & $3-\mathrm{NO}_{2}$ & $\begin{array}{c}\mathrm{C}_{37} \mathrm{H}_{26} \mathrm{~N}_{6} \mathrm{O}_{5} \mathrm{SCl}_{4} \\
(808.0)\end{array}$ & 175 & 63 & $\begin{array}{c}54.92 \\
(54.95)\end{array}$ & $\begin{array}{l}3.18 \\
(3.22)\end{array}$ & $\begin{array}{c}10.38 \\
(10.40)\end{array}$ \\
\hline $6 \mathrm{f}$. & $4-\mathrm{NO}_{2}$ & $\begin{array}{c}\mathrm{C}_{37} \mathrm{H}_{26} \mathrm{~N}_{6} \mathrm{O}_{5} \mathrm{SCl}_{4} \\
(808.0)\end{array}$ & 168 & 62 & $\begin{array}{c}54.91 \\
(54.95)\end{array}$ & $\begin{array}{c}3.18 \\
(3.22)\end{array}$ & $\begin{array}{c}10.38 \\
(10.40)\end{array}$ \\
\hline $6 \mathrm{~g}$. & $2-\mathrm{OCH}_{3}$ & $\begin{array}{c}\mathrm{C}_{38} \mathrm{H}_{29} \mathrm{~N}_{5} \mathrm{O}_{4} \mathrm{SCl}_{4} \\
(793.0)\end{array}$ & 188 & 64 & $\begin{array}{l}57.48 \\
(57.50)\end{array}$ & $\begin{array}{c}3.64 \\
(3.66)\end{array}$ & $\begin{array}{c}8.82 \\
(8.83)\end{array}$ \\
\hline $6 \mathrm{~h}$. & $\begin{array}{c}3-\mathrm{OCH}_{3}- \\
4-\mathrm{OH}\end{array}$ & $\begin{array}{c}\mathrm{C}_{38} \mathrm{H}_{29} \mathrm{~N}_{5} \mathrm{O}_{5} \mathrm{SCl}_{4} \\
(809.0)\end{array}$ & 158 & 68 & $\begin{array}{c}56.33 \\
(56.36)\end{array}$ & $\begin{array}{c}3.54 \\
(3.58)\end{array}$ & $\begin{array}{c}8.63 \\
(8.65)\end{array}$ \\
\hline $6 \mathrm{i}$. & $4-\mathrm{OCH}_{3}$ & $\begin{array}{c}\mathrm{C}_{38} \mathrm{H}_{29} \mathrm{~N}_{5} \mathrm{O}_{4} \mathrm{SCl}_{4} \\
(793.0)\end{array}$ & 182 & 60 & $\begin{array}{l}57.47 \\
(57.50)\end{array}$ & $\begin{array}{l}3.63 \\
(3.66)\end{array}$ & $\begin{array}{c}8.81 \\
(8.83)\end{array}$ \\
\hline $6 \mathrm{j}$. & $\begin{array}{l}3,4,5- \\
\left(\mathrm{OCH}_{3}\right)_{3}\end{array}$ & $\begin{array}{c}\mathrm{C}_{40} \mathrm{H}_{33} \mathrm{~N}_{5} \mathrm{O}_{6} \mathrm{SCl}_{4} \\
(853.0)\end{array}$ & 192 & 63 & $\begin{array}{c}56.24 \\
(56.27)\end{array}$ & $\begin{array}{l}3.85 \\
(3.87)\end{array}$ & $\begin{array}{c}8.18 \\
(8.21)\end{array}$ \\
\hline
\end{tabular}


Table 7. ANTIMICROBIAL DATA OF COMPOUNDS [1(a-j)]

\begin{tabular}{|c|c|c|c|c|}
\hline \multirow{2}{*}{$\begin{array}{l}\text { Compd. } \\
\text { No. }\end{array}$} & \multirow{2}{*}{$\mathrm{R}$} & \multicolumn{3}{|c|}{ Zone of Inhibition (m.m.) } \\
\hline & & $\begin{array}{c}\text { Staphylococcus aureus } \\
\text { (Antibacterial) }\end{array}$ & $\begin{array}{c}\text { Escherichia coli } \\
\text { (Antibacterial) }\end{array}$ & $\begin{array}{l}\text { Candela albicans } \\
\text { (Antifungal) }\end{array}$ \\
\hline 1 a. & $2-\mathrm{OH}$ & 12.0 & 10.0 & 8.0 \\
\hline $1 \mathrm{~b}$. & 2-CI & 10.0 & 9.0 & 7.0 \\
\hline $1 \mathrm{c}$. & $3-\mathrm{CI}$ & 9.0 & 9.0 & 5.0 \\
\hline $1 \mathrm{~d}$. & $2-\mathrm{NO}_{2}$ & 12.0 & 11.0 & 7.0 \\
\hline $1 \mathrm{e}$. & $3-\mathrm{NO}_{2}$ & 11.0 & 10.0 & 6.0 \\
\hline $1 \mathrm{f}$. & $4-\mathrm{NO}_{2}$ & 8.0 & 9.0 & 4.0 \\
\hline $1 \mathrm{~g}$. & $2-\mathrm{OCH}_{3}$ & 9.0 & 8.0 & 5.0 \\
\hline $1 \mathrm{~h}$. & 3-OCH3-4-OH & 11.0 & 10.0 & 7.0 \\
\hline $1 \mathrm{i}$. & $4-\mathrm{OCH}_{3}$ & 10.0 & 9.0 & 6.0 \\
\hline $1 \mathrm{j}$. & $3,4,5-\left(\mathrm{OCH}_{3}\right)_{3}$ & 11.0 & 12.0 & 8.0 \\
\hline
\end{tabular}

Table 8. ANTIMICROBIAL DATA OF COMPOUNDS [2(a-j)]

\begin{tabular}{ccccc}
\hline \multirow{2}{*}{$\begin{array}{c}\text { Compd. } \\
\text { No. }\end{array}$} & $\mathrm{R}$ & \multicolumn{3}{c}{ Zone of Inhibition (m.m.) } \\
\cline { 3 - 5 } & & $\begin{array}{c}\text { Staphylococcus aureus } \\
\text { (Antibacterial) }\end{array}$ & $\begin{array}{c}\text { Escherichia coli } \\
\text { (Antibacterial) }\end{array}$ & $\begin{array}{c}\text { Candela albicans } \\
\text { (Antifungal) }\end{array}$ \\
\hline 2 a. & $2-\mathrm{OH}$ & 10.0 & 10.0 & 7.0 \\
2 b. & $2-\mathrm{CI}$ & 8.0 & 9.0 & 5.0 \\
2 c. & $3-\mathrm{CI}$ & 9.0 & 8.0 & 4.0 \\
2 d. & $2-\mathrm{NO}_{2}$ & 11.0 & 10.0 & 7.0 \\
2 e. & $3-\mathrm{NO}_{2}$ & 10.0 & 12.0 & 8.0 \\
2 f. & $4-\mathrm{NO}_{2}$ & 8.0 & 9.0 & 5.0 \\
2 g. & $2-\mathrm{OCH}_{3}$ & 11.0 & 9.0 & 6.0 \\
2 h. & $3-\mathrm{OCH}_{3}-4-\mathrm{OH}$ & 12.0 & 12.0 & 8.0 \\
2 i. & $4-\mathrm{OCH}_{3}$ & 9.0 & 7.0 & 6.0 \\
2 j. & $3,4,5-\left(\mathrm{OCH}_{3}\right)_{3}$ & 10.0 & 11.0 & 7.0 \\
\hline
\end{tabular}


Table 9. ANTIMICROBIAL DATA OF COMPOUNDS [3(a-j)]

\begin{tabular}{|c|c|c|c|c|}
\hline \multirow{2}{*}{$\begin{array}{l}\text { Compd. } \\
\text { No. }\end{array}$} & \multirow[b]{2}{*}{$\mathrm{R}$} & \multicolumn{3}{|c|}{ Zone of Inhibition (m.m.) } \\
\hline & & $\begin{array}{c}\text { Staphylococcus aureus } \\
\text { (Antibacterial) }\end{array}$ & $\begin{array}{c}\text { Escherichia coli } \\
\text { (Antibacterial) }\end{array}$ & $\begin{array}{c}\text { Candela albicans } \\
\text { (Antifungal) }\end{array}$ \\
\hline 3 a. & $2-\mathrm{OH}$ & 9.0 & 10.0 & 7.0 \\
\hline $3 \mathrm{~b}$. & $2-\mathrm{CI}$ & 10.0 & 8.0 & 6.0 \\
\hline $3 \mathrm{c}$. & $3-\mathrm{CI}$ & 7.0 & 7.0 & 4.0 \\
\hline $3 \mathrm{~d}$ & $2-\mathrm{NO}_{2}$ & 11.0 & 12.0 & 8.0 \\
\hline 3 e. & $3-\mathrm{NO}_{2}$ & 10.0 & 11.0 & 6.0 \\
\hline $3 \mathrm{f}$. & $4-\mathrm{NO}_{2}$ & 8.0 & 9.0 & 5.0 \\
\hline $3 \mathrm{~g}$. & $2-\mathrm{OCH}_{3}$ & 12.0 & 10.0 & 8.0 \\
\hline $3 \mathrm{~h}$. & $3-\mathrm{OCH}_{3}-4-\mathrm{OH}$ & 11.0 & 12.0 & 7.0 \\
\hline $3 \mathrm{i}$. & $4-\mathrm{OCH}_{3}$ & 8.0 & 7.0 & 4.0 \\
\hline $3 \mathrm{j}$. & $3,4,5-\left(\mathrm{OCH}_{3}\right)_{3}$ & 9.0 & 7.0 & 5.0 \\
\hline
\end{tabular}

Table 10. ANTIMICROBIAL DATA OF COMPOUNDS [4(a-j)]

\begin{tabular}{ccccc}
\hline \multirow{2}{*}{$\begin{array}{c}\text { Compd. } \\
\text { No. }\end{array}$} & $\mathrm{R}$ & \multicolumn{3}{c}{ Zone of Inhibition (m.m.) } \\
\cline { 3 - 5 } & & $\begin{array}{c}\text { Staphylococcus aureus } \\
\text { (Antibacterial) }\end{array}$ & $\begin{array}{c}\text { Escherichia coli } \\
\text { (Antibacterial) }\end{array}$ & $\begin{array}{c}\text { Candela albicans } \\
\text { (Antifungal) }\end{array}$ \\
\hline 4 a. & $2-\mathrm{OH}$ & 12.0 & 11.0 & 8.0 \\
4 b. & $2-\mathrm{CI}$ & 9.0 & 9.0 & 5.0 \\
$4 \mathrm{c}$. & $3-\mathrm{CI}$ & 7.0 & 8.0 & 4.0 \\
$4 \mathrm{~d}$. & $2-\mathrm{NO}_{2}$ & 11.0 & 10.0 & 6.0 \\
4 e. & $3-\mathrm{NO}_{2}$ & 12.0 & 10.0 & 7.0 \\
4 f. & $4-\mathrm{NO}_{2}$ & 8.0 & 11.0 & 6.0 \\
4 g. & $2-\mathrm{OCH}_{3}$ & 11.0 & 12.0 & 8.0 \\
4 h. & $3-\mathrm{CH}_{3}-4-\mathrm{OH}$ & 11.0 & 10.0 & 7.0 \\
4 i. & $4-\mathrm{OCH}_{3}$ & 9.0 & 9.0 & 6.0 \\
4 j. & $3,4,5-\left(\mathrm{OCH}_{3}\right)_{3}$ & 11.0 & 12.0 & 8.0 \\
\hline
\end{tabular}


Table 11.ANTIMICROBIAL DATA OF COMPOUNDS [5(a-j)]

\begin{tabular}{ccccc}
$\begin{array}{c}\text { Compd. } \\
\text { No. }\end{array}$ & $\mathrm{R}$ & \multicolumn{3}{c}{ Zone of Inhibition (m.m.) } \\
\cline { 3 - 5 } & & $\begin{array}{c}\text { Staphylococcus aureus } \\
\text { (Antibacterial) }\end{array}$ & $\begin{array}{c}\text { Escherichia coli } \\
\text { (Antibacterial) }\end{array}$ & $\begin{array}{c}\text { Candela albicans } \\
\text { (Antifungal) }\end{array}$ \\
\hline 5 a. & $2-\mathrm{OH}$ & 12.0 & 12.0 & 8.0 \\
5 b. & $2-\mathrm{CI}$ & 9.0 & 10.0 & 5.0 \\
5 c. & $3-\mathrm{CI}$ & 10.0 & 12.0 & 7.0 \\
5 d. & $2-\mathrm{NO}_{2}$ & 11.0 & 9.0 & 7.0 \\
5 e. & $3-\mathrm{NO}_{2}$ & 10.0 & 8.0 & 6.0 \\
5 f. & $4-\mathrm{NO}_{2}$ & 12.0 & 10.0 & 8.0 \\
5 g. & $2-\mathrm{OCH}_{3}$ & 8.0 & 8.0 & 4.0 \\
5 h. & $3-\mathrm{OCH}_{3}-4-\mathrm{OH}$ & 11.0 & 10.0 & 7.0 \\
5 i. & $4-\mathrm{OCH}_{3}$ & 9.0 & 11.0 & 6.0 \\
5 j. & $3,4,5-\left(\mathrm{OCH}_{3}\right)_{3}$ & 8.0 & 7.0 & 4.0 \\
\hline
\end{tabular}

Table 12. ANTIMICROBIAL DATA OF COMPOUNDS [6(a-j)]

\begin{tabular}{ccccc}
\hline \multirow{2}{*}{$\begin{array}{c}\text { Nompd. } \\
\text { No. }\end{array}$} & $\mathrm{R}$ & $\begin{array}{c}\text { Zone of Inhibition (m.m.) } \\
\text { (Antibacterial) }\end{array}$ & $\begin{array}{c}\text { Escherichia coli } \\
\text { (Antibacterial) }\end{array}$ & $\begin{array}{c}\text { Candela albicans } \\
\text { (Antifungal) }\end{array}$ \\
\hline 6 a. & $2-\mathrm{OH}$ & 11.0 & 12.0 & 8.0 \\
6 b. & $2-\mathrm{CI}$ & 7.0 & 9.0 & 4.0 \\
6 c. & $3-\mathrm{CI}$ & 9.0 & 10.0 & 6.0 \\
6 d. & $2-\mathrm{NO}_{2}$ & 11.0 & 8.0 & 7.0 \\
6 e. & $3-\mathrm{NO}_{2}$ & 12.0 & 11.0 & 8.0 \\
6 f. & $4-\mathrm{NO}_{2}$ & 10.0 & 10.0 & 6.0 \\
6 g. & $2-\mathrm{OCH}_{3}$ & 12.0 & 10.0 & 8.0 \\
6 h. & $3-\mathrm{OCH}_{3}-4-\mathrm{OH}$ & 9.0 & 11.0 & 7.0 \\
6 i. & $4-\mathrm{OCH}_{3}$ & 8.0 & 7.0 & 5.0 \\
6 j. & $3,4,5-\left(\mathrm{OCH}_{3}\right)_{3}$ & 10.0 & 8.0 & 6.0 \\
\hline
\end{tabular}




\section{Materials and Methods}

All melting points were determined in open capillaries in a liquid paraffin bath and are uncorrected. The I. R. spectra were recorded with $\mathrm{KBr}$ pellets on Perkin - Elmer - 783 spectrophotometer and ${ }^{1} \mathrm{H}$ N.M.R. spectra were recorded on a Varian Geminy $200 \mathrm{MHz}$ spectrophotometer with $\mathrm{CDCl}_{3} / \mathrm{DMSO}$ $\mathrm{d}_{6}$ as a solvent using tetramethylsilane (T.M.S.) as an internal standard; the chemical shift values are in $\delta \mathrm{ppm}$. The purity of the compounds was checked by thin layer chromatography (T.L.C.) on silica gel coated glass plates. The elemental analysis (i.e. C, $\mathrm{H}$ and $\mathrm{N}$ analysis) has been done on Carlo - Erba 1108 analyzer and the values are within the permissible limits (i.e. \pm 0.5 ) of their calculated values.

\section{Antimicrobial activity}

Antimicrobial activity of newly synthesised compounds was studied against gram-positive bacteria "Staphylococcus aureus" and gram-negative bacteria "Escherichia coli" (for antibacterial activity) and against the culture "Candela albicans" (for antifungal activity). The antimicrobial screening was carried out by cup - plate method ${ }^{10}$ at a concentration of $50 \mu \mathrm{g} / \mathrm{mL}$ in solvent D.M.F. The zone of inhibition was measured in $\mathrm{mm}$. The antimicrobial activity of the synthesised compounds was compared with standard drugs Ampicillin, Penicillin and Tetracycline at the same concentration.

\section{Results and Discussion}

The antimicrobial activities of newly synthesised compounds were compared with known antibiotics like Ampicillin, Penicillin and Tetracycline and all the compounds show moderate to good activity. Structure elucidation of synthesised compounds has been made on the basis of elemental analysis, I.R. spectral studies and ${ }^{1}$ H N.M.R. spectral studies and all the compounds gave satisfactory elemental analysis, I.R. and ${ }^{1}$ H N.M.R. spectral measurements.

\section{I.R. Spectral Studies}

\section{$>\quad$ I.R. $\left(\mathrm{cm}^{-1}\right)(\mathrm{KBr})$ spectral data of compound [1(e)] :-}

$1662 \vee(\mathrm{C}=\mathrm{O}$ stretching, chalcone moiety); $1604 \vee(\mathrm{C}=\mathrm{N}$ stretching, pyrazoline moiety); $1585 \vee(\mathrm{C}=\mathrm{C}$ stretching, chalcone moiety); $1526 \mathrm{v}\left(\mathrm{N}=\mathrm{O}\right.$ stretching, $\mathrm{Ar}-\mathrm{NO}_{2}$ at phenyl ring of chalcone moiety); $1474 \mathrm{v}\left(\mathrm{C}-\mathrm{H}\right.$ bending, $-\mathrm{CH}_{2^{-}}$of pyrazoline ring); $1348 \vee\left(\mathrm{S}=\mathrm{O}\right.$ stretching, $\left.\mathrm{Ar}-\mathrm{SO}_{2} \mathrm{NH}-\mathrm{Ar}\right) ; 735 \mathrm{v}$ (C-Cl stretching, $\mathrm{Ar}-\mathrm{Cl}$ at phenyl ring).

$>\quad$ I.R. $\left(\mathrm{cm}^{-1}\right)(\mathrm{KBr})$ spectral data of compound [2(b)] :-

$3400 \vee(\mathrm{N}-\mathrm{H}$ stretching, pyrimidine moiety); $1658 \vee(\mathrm{C}=\mathrm{O}$ stretching, pyrimidine moiety); $1600 \mathrm{v}$ $\left(\mathrm{C}=\mathrm{N}\right.$ stretching, pyrazoline moiety); $1465 \vee\left(\mathrm{C}-\mathrm{H}\right.$ bending, $-\mathrm{CH}_{2^{-}}$of pyrazoline ring); $1340 \vee(\mathrm{S}=\mathrm{O}$ stretching, $\left.\mathrm{Ar}_{-} \mathrm{SO}_{2} \mathrm{NH}-\mathrm{Ar}\right) ; 745 \mathrm{v}(\mathrm{C}-\mathrm{Cl}$ stretching, $\mathrm{Ar}-\mathrm{Cl}$ at phenyl ring).

\section{$>\quad$ I.R. $\left(\mathrm{cm}^{-1}\right)(\mathrm{KBr})$ spectral data of compound [3(i)] :-}

$3367 v\left(\mathrm{~N}-\mathrm{H}\right.$ stretching, pyrimidine moiety); $2833 v$ (C-H stretching, $\mathrm{Ar}-\mathrm{OCH}_{3}$ at phenyl ring); $1604 \mathrm{v}$ $\left(\mathrm{C}=\mathrm{N}\right.$ stretching, pyrazoline moiety); $1471 \vee\left(\mathrm{C}-\mathrm{H}\right.$ bending, $-\mathrm{CH}_{2}$ - of pyrazoline ring); $1352 \vee(\mathrm{S}=\mathrm{O}$ stretching, $\left.\mathrm{Ar}_{-} \mathrm{SO}_{2} \mathrm{NH}-\mathrm{Ar}\right) ; 1198 \vee(\mathrm{C}=\mathrm{S}$ stretching, pyrimidine moiety); $736 v(\mathrm{C}-\mathrm{Cl}$ stretching, $\mathrm{Ar}-\mathrm{Cl}$ at phenyl ring).

$>\quad$ I.R. $\left(\mathrm{cm}^{-1}\right)(\mathrm{KBr})$ spectral data of compound [4(c)] :-

$3400 \vee(\mathrm{N}-\mathrm{H}$ stretching, pyrazoline moiety); $1610 \vee(\mathrm{C}=\mathrm{N}$ stretching, pyrazoline moiety); $1468 \vee(\mathrm{C}-\mathrm{H}$ bending, $-\mathrm{CH}_{2}$ - of pyrazoline ring); $1345 \vee\left(\mathrm{S}=\mathrm{O}\right.$ stretching, $\left.\mathrm{Ar}_{-} \mathrm{SO}_{2} \mathrm{NH}-\mathrm{Ar}\right) ; 750 \vee(\mathrm{C}-\mathrm{Cl}$ stretching, $\mathrm{Ar}-\mathrm{Cl}$ at phenyl ring).

$>\quad$ I.R. $\left(\mathrm{cm}^{-1}\right)(\mathrm{KBr})$ spectral data of compound [5(j)] :-

$3380 \vee(\mathrm{N}-\mathrm{H}$ stretching, Ar-NH-CO-CH$) ; 2964 v\left(\mathrm{C}-\mathrm{H}\right.$ stretching, $\left.-\mathrm{CO}-\mathrm{CH}_{3}\right) ; 2832 \vee(\mathrm{C}-\mathrm{H}$ stretching, $\mathrm{Ar}-\mathrm{OCH}_{3}$ at phenyl ring $) ; 1657 \vee(\mathrm{C}=\mathrm{O}$ stretching, $-\mathrm{NH}-\mathrm{CO}-) ; 1606 \vee(\mathrm{C}=\mathrm{N}$ stretching, pyrazoline moiety); $1450 \vee\left(\mathrm{C}-\mathrm{H}\right.$ bending, $-\mathrm{CH}_{2^{-}}$of pyrazoline ring); $1348 \vee\left(\mathrm{S}=\mathrm{O}\right.$ stretching, $\left.\mathrm{Ar}-\mathrm{SO}_{2} \mathrm{NH}-\mathrm{Ar}\right)$; $1170 \vee\left(\mathrm{S}=\mathrm{O}\right.$ stretching, $\left.\mathrm{Ar}_{-} \mathrm{SO}_{2}-\mathrm{Ar}\right) ; 745 \mathrm{v}(\mathrm{C}-\mathrm{Cl}$ stretching, $\mathrm{Ar}-\mathrm{Cl}$ at phenyl ring).

$>\quad$ I.R. $\left(\mathrm{cm}^{-1}\right)(\mathrm{KBr})$ spectral data of compound $[6(\mathrm{~g})]:-$

$2834 \vee\left(\mathrm{C}-\mathrm{H}\right.$ stretching, $\mathrm{Ar}-\mathrm{OCH}_{3}$ at phenyl ring); $1665 \vee(\mathrm{C}=\mathrm{O}$ stretching, $\mathrm{Ar}-\mathrm{CO}-\mathrm{Ar}) ; 1610 \vee(\mathrm{C}=\mathrm{N}$ stretching, pyrazoline moiety); $1462 \vee\left(\mathrm{C}-\mathrm{H}\right.$ bending, $-\mathrm{CH}_{2^{-}}$of pyrazoline ring); $1352 \vee(\mathrm{S}=\mathrm{O}$ stretching, $\left.\mathrm{Ar}_{-} \mathrm{SO}_{2} \mathrm{NH}-\mathrm{Ar}\right) ; 736 \vee(\mathrm{C}-\mathrm{Cl}$ stretching, $\mathrm{Ar}-\mathrm{Cl}$ at phenyl ring). 


\section{${ }^{1}$ H N.M.R. Spectral Studies}

$>{ }^{1} \mathrm{H}$ N.M.R. $\left(\mathrm{CDCl}_{3}\right)$ spectral data of compound [1(f)] :-

$3.30 \delta \mathrm{ppm}\left(\mathrm{s}, 2 \mathrm{H},-\mathrm{CH}_{2^{-}}\right.$of pyrazoline ring); $3.38 \delta \mathrm{ppm}(\mathrm{s}, 1 \mathrm{H}, \mathrm{Ar}-\mathrm{CH}) ; 7.03$ to $7.75 \delta \mathrm{ppm}(\mathrm{m}$, $14 \mathrm{H}, \operatorname{Ar}-\mathrm{H}) ; 7.79 \delta \mathrm{ppm}(\mathrm{d}, 1 \mathrm{H},-\mathrm{CH}=\mathrm{CH}-\mathrm{Ar}) ; 8.14 \delta \mathrm{ppm}(\mathrm{d}, 1 \mathrm{H},-\mathrm{CO}-\mathrm{CH}=\mathrm{CH}-) ; 8.22 \delta$ $\operatorname{ppm}\left(\mathrm{s}, 1 \mathrm{H}, \mathrm{Ar}-\mathrm{SO}_{2} \mathrm{NH}-\mathrm{Ar}\right)$.

$>{ }^{1} \mathrm{H}$ N.M.R. $\left(\mathrm{CDCl}_{3}\right)$ spectral data of compound [2(i)] :-

$3.27 \delta \mathrm{ppm}\left(\mathrm{s}, 2 \mathrm{H},-\mathrm{CH}_{2}\right.$ - of pyrazoline ring); $3.35 \delta \mathrm{ppm}\left(\mathrm{s}, 2 \mathrm{H},-\mathrm{CH}_{2}\right.$ - of pyrimidine ring); $3.41 \delta$ $\mathrm{ppm}(\mathrm{s}, 1 \mathrm{H}, \quad \mathrm{Ar}-\mathrm{CH}) ; 3.78 \delta \mathrm{ppm}\left(\mathrm{s}, 3 \mathrm{H}, \mathrm{Ar}-\mathrm{OCH}_{3}\right.$ at phenyl ring); 7.01 to $7.71 \delta \mathrm{ppm}(\mathrm{m}, 14 \mathrm{H}$, $\mathrm{Ar}-\mathrm{H}) ; 7.84 \delta \mathrm{ppm}\left(\mathrm{s}, 1 \mathrm{H},-\mathrm{NH}\right.$ - of pyrimidine ring); $8.24 \delta \mathrm{ppm}\left(\mathrm{s}, 1 \mathrm{H}, \mathrm{Ar}_{-} \mathrm{SO}_{2} \mathrm{NH}-\mathrm{Ar}\right)$.

$>{ }^{1} \mathrm{H}$ N.M.R. $\left(\mathrm{CDCl}_{3}\right)$ spectral data of compound [3(g)] :-

$3.28 \delta \mathrm{ppm}\left(\mathrm{s}, 2 \mathrm{H},-\mathrm{CH}_{2^{-}}\right.$of pyrazoline ring); $3.33 \delta \mathrm{ppm}\left(\mathrm{s}, 2 \mathrm{H},-\mathrm{CH}_{2^{-}}\right.$of pyrimidine ring); $3.40 \delta$ ppm (s, $1 \mathrm{H}, \quad \mathrm{Ar}-\mathrm{CH}) ; 3.80 \delta \mathrm{ppm}\left(\mathrm{s}, 3 \mathrm{H}, \mathrm{Ar}-\mathrm{OCH}_{3}\right.$ at phenyl ring); 6.99 to $7.68 \delta \mathrm{ppm}(\mathrm{m}, 14 \mathrm{H}$, $\mathrm{Ar}-\mathrm{H}) ; 7.83 \delta \mathrm{ppm}\left(\mathrm{s}, 1 \mathrm{H},-\mathrm{NH}\right.$ - of pyrimidine ring); $8.20 \delta \mathrm{ppm}\left(\mathrm{s}, 1 \mathrm{H}, \mathrm{Ar}_{-} \mathrm{SO}_{2} \mathrm{NH}-\mathrm{Ar}\right.$ ).

$>{ }^{1} \mathrm{H}$ N.M.R. $\left(\mathrm{CDCl}_{3}+\mathrm{DMSO}_{-} \mathrm{d}_{6}\right)$ spectral data of compound $[4(\mathrm{~b})]$ :-

$3.31 \delta \mathrm{ppm}\left(\mathrm{s}, 4 \mathrm{H}, 2 \times-\mathrm{CH}_{2}\right.$ - of pyrazoline ring); $3.39 \delta \mathrm{ppm}(\mathrm{s}, 1 \mathrm{H}, \mathrm{Ar}-\mathrm{CH}) ; 7.05$ to $7.72 \delta \mathrm{ppm}(\mathrm{m}$, $14 \mathrm{H}, \mathrm{Ar}-\mathrm{H}) ; 7.80 \delta \mathrm{ppm}\left(\mathrm{s}, 1 \mathrm{H},-\mathrm{NH}\right.$ - of pyrazoline ring); $8.21 \delta \mathrm{ppm}\left(\mathrm{s}, 1 \mathrm{H}, \mathrm{Ar}_{-} \mathrm{SO}_{2} \mathrm{NH}-\mathrm{Ar}\right)$.

$>{ }^{1} \mathrm{H}$ N.M.R. $\left(\mathrm{CDCl}_{3}\right)$ spectral data of compound $[\mathbf{5}(\mathrm{j})]$ :-

$3.33 \delta \mathrm{ppm}\left(\mathrm{s}, 4 \mathrm{H}, 2 \times-\mathrm{CH}_{2}\right.$ - of pyrazoline ring); $3.42 \delta \mathrm{ppm}(\mathrm{s}, 1 \mathrm{H}, \mathrm{Ar}-\mathrm{CH}) ; 3.60 \delta \mathrm{ppm}(\mathrm{s}, 3 \mathrm{H},-$ $\mathrm{CO}-\mathrm{CH}_{3}$ ); $3.79 \delta \mathrm{ppm}$ (s, $6 \mathrm{H}, \mathrm{Ar}-\mathrm{OCH}_{3}$ at phenyl ring); $3.83 \delta \mathrm{ppm}, 3.85 \delta \mathrm{ppm}$ (s each, $3 \mathrm{H}, 2 \times \mathrm{Ar}-$ $\mathrm{OCH}_{3}$ at phenyl ring); $5.71 \delta \mathrm{ppm}(\mathrm{s}, 1 \mathrm{H}, \mathrm{Ar}-\mathrm{NH}-\mathrm{CO}-) ; 7.04$ to $7.76 \delta \mathrm{ppm}(\mathrm{m}, 16 \mathrm{H}, \mathrm{Ar}-\mathrm{H})$; $8.22 \delta \mathrm{ppm}\left(\mathrm{s}, 1 \mathrm{H}, \mathrm{Ar}-\mathrm{SO}_{2} \mathrm{NH}-\mathrm{Ar}\right)$.

$>{ }^{1} \mathrm{H}$ N.M.R. $\left(\mathrm{CDCl}_{3}+\right.$ DMSO - $\left.\mathrm{d}_{6}\right)$ spectral data of compound [6(d)] :-

$3.32 \delta \mathrm{ppm}\left(\mathrm{s}, 4 \mathrm{H}, 2 \times-\mathrm{CH}_{2}\right.$ - of pyrazoline ring); $3.44 \delta \mathrm{ppm}(\mathrm{s}, 1 \mathrm{H}, \mathrm{Ar}-\mathrm{CH}) ; 7.00$ to $7.78 \delta \mathrm{ppm}(\mathrm{m}$, $19 \mathrm{H}, \mathrm{Ar}-\mathrm{H}) ; 8.19 \delta \mathrm{ppm}\left(\mathrm{s}, 1 \mathrm{H}, \mathrm{Ar}_{-} \mathrm{SO}_{2} \mathrm{NH}-\mathrm{Ar}\right)$.

\section{Acknowledgements}

The authors are thankful to the Department of Chemistry, Veer Narmad South Gujarat University, Surat, for providing necessary laboratory facilities. They are also grateful to the Department of Biosciences, Veer Narmad South Gujarat University, Surat, for screening the newly synthesised compounds for their antimicrobial activities; Wyeth Lederle Limited, Atul, for scanning the I.R. spectra and Department of Central Instrumentation Laboratory, Punjab University, Chandigarh, for screening the ${ }^{1}$ H N.M.R. spectra of newly synthesised compounds.

\section{References}

1. $\quad$ Mehta K J, Patel V S and Parikh A R, J Indian Chem Soc, 1978, 50, 241.

2. Mudaliar V and Joshi V, Indian J Chem, 1995, 34B, 456.

3. Hosni G and Saad S F, Acta Chim Acad Sci Hung, 1995, 86, 263.

4. Hishmat O H, El-Diwani H I and Melek F R, Indian J Chem, 1996, 35B, 30.

5. Hollis H D, Johnson J L, Werbel L M, Leopold W R, Jackson R C and Elslager E F, J Med Chem, 1984, 27, 253.

6. Grosscurt A C, Van Hes R and Wellinga K, J Agric Food Chem, 1979, 27, 406.

7. Barot V M., Asian J Chem, 1996, 8, 565.

8. El-Hashash M A, Mahmoud M R and Madboli S A, Indian J Chem, 1993, 32B, 449.

9. Calabresi P and Parks R E (Jr.) in: Goodman L S and Gilman A (Eds.); The Pharmacological Basis of Therapeutics, Macmillan, New York, 1975, $5^{\text {th }}$ Edn., p.1254.

10. Barry A L, in Illus (Ed.), The Antimicrobial Susceptibility Test : Principle and Practices, Lea and Febiger, Philadelphia, Pa, U.S.A., 1976, p.180; Biol. Abstr., 1977, 64, 25183. 


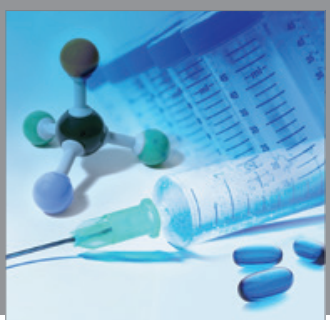

International Journal of

Medicinal Chemistry

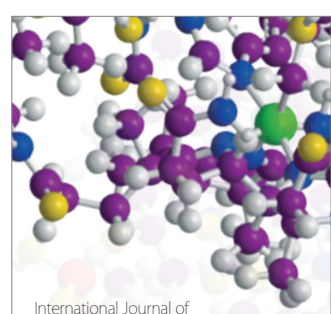

Carbohydrate Chemistry

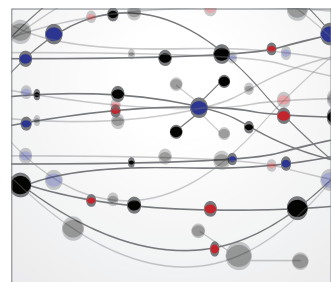

The Scientific World Journal
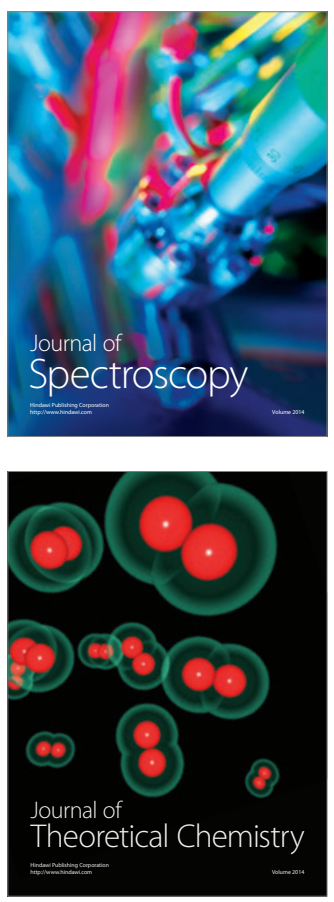
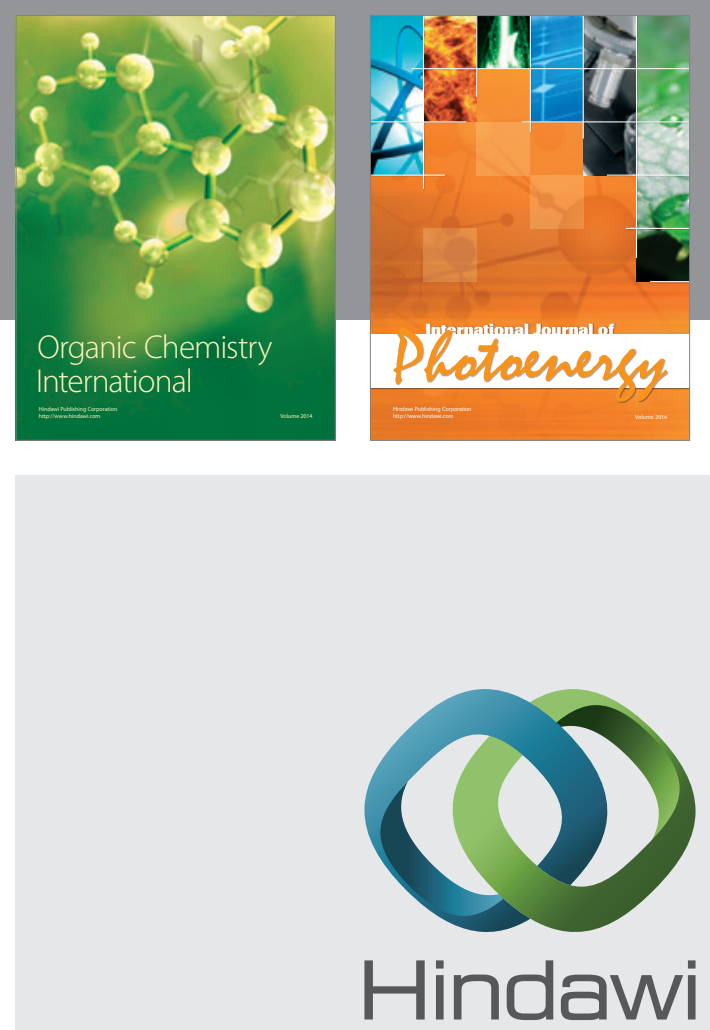

Submit your manuscripts at

http://www.hindawi.com
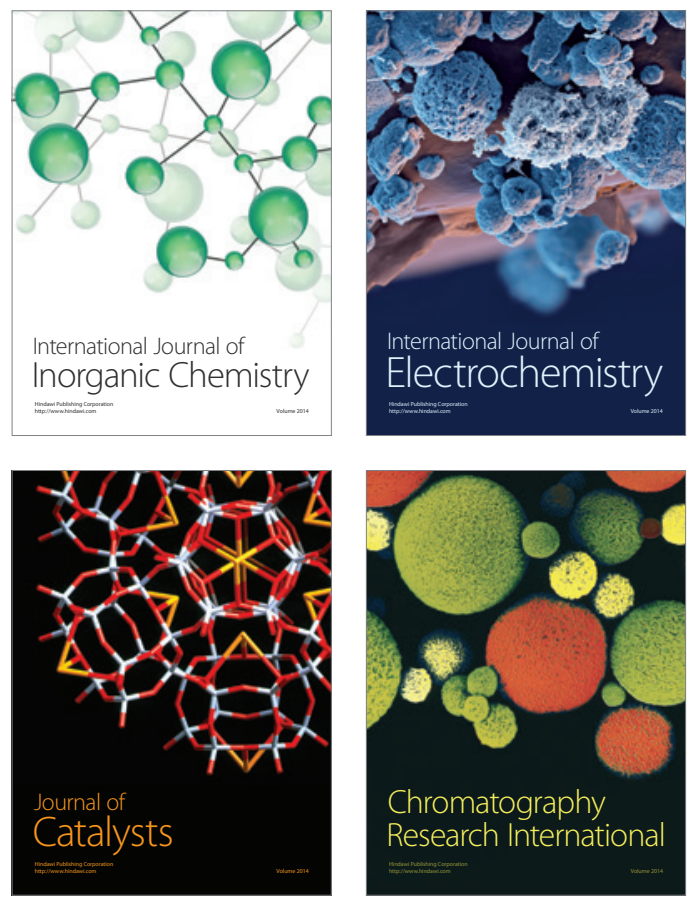
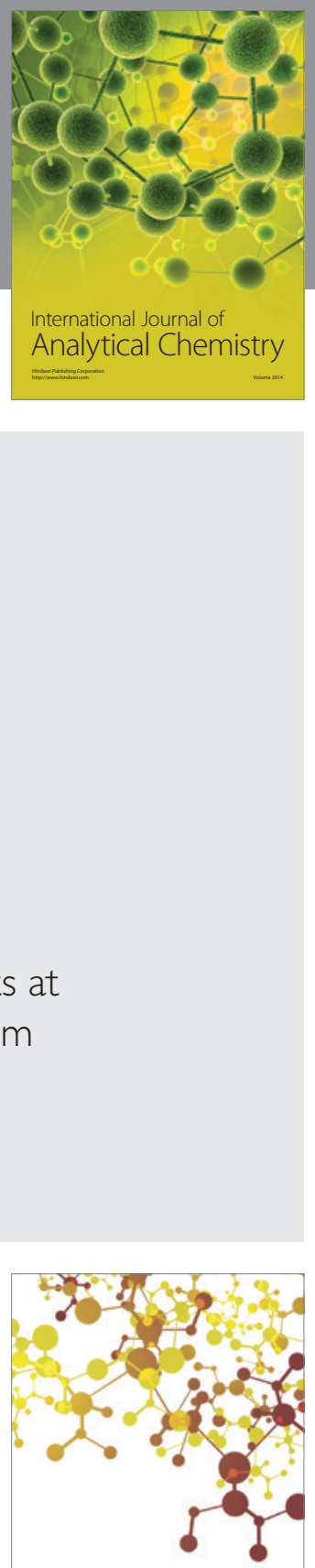

Journal of

Applied Chemistry
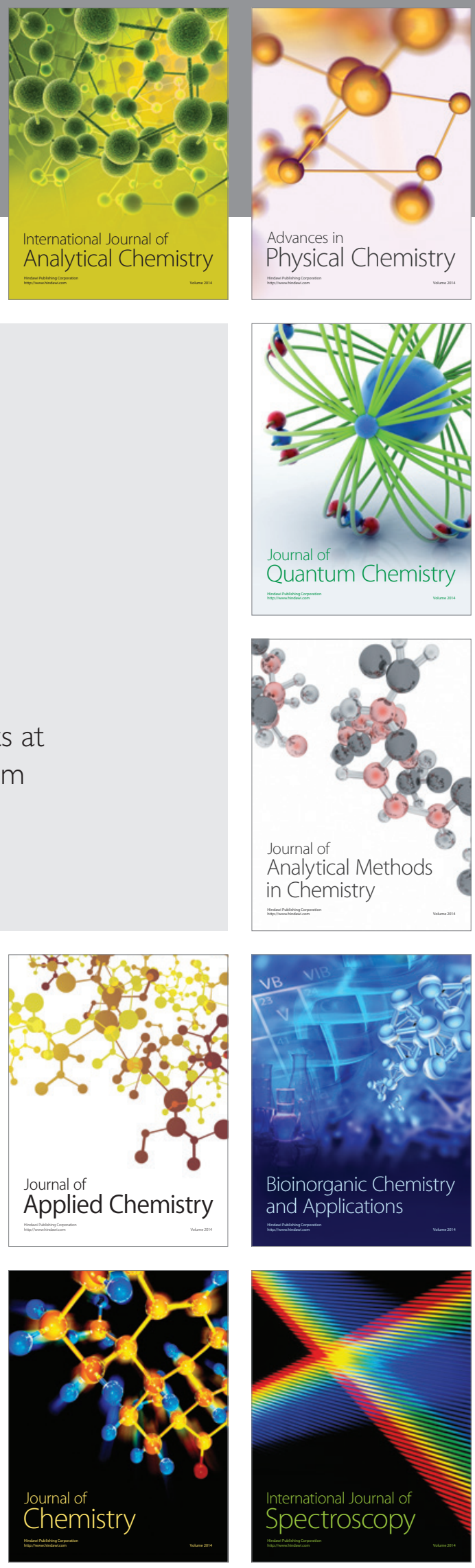